\title{
On the formation and morphology of coherent particulate structures in non-isothermal enclosures subjected to rotating g-jitters
}

\author{
Marcello Lappa \\ Department of Mechanical and Aerospace Engineering, University of Strathclyde \\ James Weir Building, 75 Montrose Street, Glasgow, G1 1XJ, UK \\ email: marcello.lappa@strath.ac.uk
}

\begin{abstract}
:
The strategy undertaken in earlier author's work (M. Lappa, Phys. Fluids, 26(9), 093301, 2014; M. Lappa, Int. J. Multiphase Flow, 93: 71-83, 2017) based on the use of polarized (purely translational) vibrations for achieving the segregation or accumulation of solid particles in specific regions of an initially dilute dispersion is further pursued by allowing the direction of vibrations to change in time with respect to the applied temperature difference. In particular, the potential of the considered approach in separating the particles from the liquid is explored under the assumption that the angular velocity by which the vibrations axis rotates about a fixed axis is of the same order of magnitude or smaller (one or two orders of magnitude) than the frequency of shaking. A new family of particle coherent structures is identified in the physical space, which can be distinguished from the companion category of particle attractors for fixed vibration direction due to its increased symmetry properties. It is shown how the average nonlinear effects produced by the rotation of the vibration axis, together with those induced by the finite size of the enclosure, accumulate over time leading to the observed fascinating variety of symmetrical patterns.
\end{abstract}

Keywords: Particle segregation and accumulation, thermovibrational flow.

\section{Introduction}

Diffusion, convective transport, dispersal and aggregation of small particles in fluid flow are mechanisms playing an overarching role in a variety of natural phenomena and technological applications (see, e.g., Melnikov and Shevtsova ${ }^{1}$; Saghir and Mohamed ${ }^{2}$; Balboa-Usabiaga and Delgado-Buscalioni ${ }^{3}$; Gotoda et al., ${ }^{4}$; Haeri ${ }^{5}$; Delgado-Buscalioni e al., ${ }^{6}$; Esmaeeli ${ }^{7}$; Lappa ${ }^{8,9}$ and Lappa et al., ${ }^{10}$; and references therein). Related processes can involve relatively wide intervals of length and time scales (ranging from microns to millimetres and seconds to hours, respectively, in typical industrial methods dealing with colloidal suspensions or powder processing).

In such a context, the possibility to induce the segregation of particles in specific regions of the physical domain is generally regarded as a topic of great importance due to the expected strong technological impact enabled accordingly.

Segregation is a counterintuitive phenomenon by which particles initially dispersed more or less uniformly in a liquid, separate from the hosting medium under some "forcing" or "excitation". Such 
phenomena are not only of great practical importance due to the aforementioned existing industrial processes but also because improving our understanding of the rich contextual information associated with them, will help us to elaborate new applications and perhaps build up completely new technological domains. Among the modern ideas currently pursued along these lines, we may mention concepts such as "deterministic hydrodynamics", "inertial clustering", the use of electric or magnetic fields or other specific body forces.

As an example of "deterministic hydrodynamics" and related applications the reader may consider the transport of suspended particles in a two-dimensional (2D) periodic lattice of obstacles (Balvin et al., ${ }^{11}$ ). This specific subject has recently attracted significant attention as a means for separating particle species in microfluidic devices. Particles are forced to move through the system by a driving force. Depending on the properties of the particles and the direction of the driving force with respect to the lattice orientation, the particles move through the lattice in commensurate phaselocked trajectories.

Another well-documented phenomenon that can give rise to fascinating particle dynamics in a fluid is inertial clustering, i.e. concentration of particles into narrow regions due to their inertia. Several studies pertaining to this line of inquiry (see, e.g., Melnikov et al., ${ }^{12}$; Gotoda et al., ${ }^{4,13}$; Melnikov and Shevtsova ${ }^{1}$ for some recent examples) have shown both theoretically and experimentally that the dynamics of a particle or a droplet with nonzero mass and size can deviate significantly with respect to that of an ideal point-like tracer with no mass and spatial extension. The physical principle governing such behaviours is that, because particles have a finite inertia, their velocity field can violate the incompressibility constraint that would be typical of a pure liquid and other incompressible flows (see, e.g., Balboa Usubiaga et al., ${ }^{14}$ ). From a mathematical point of view, this is equivalent to stating that the velocity field of particles is not solenoidal or divergence-free. For such conditions, it has been demonstrated that dispersed matter can form clusters or accumulation structures, which are not possible for the incompressible motion of infinitesimal particles. Some interesting arguments and precise criteria along these lines have been elaborated by Haller and Sapsis ${ }^{15}$ and Sapsis and Haller ${ }^{16}$.

This line of research has been recently expanded by addressing the role played in this context by mechanisms of phase-locking between traveling fluid-dynamic disturbances (propagating in the considered fluid medium in the form of waves of given wavenumber and frequency) on the one hand, and the topological and spatio-temporal properties of the particle trajectory, on the other hand (an example being the characteristic frequency taken by the particle to follow a given closed path, Schwabe et al et al., ${ }^{17}$, Pushkin et al., ${ }^{18}$ or its angular $\operatorname{spin}^{19-21}$ ). Related ideas have their root in typical concepts of deterministic hydrodynamics (as discussed above), in which the components being fractionated become locked into specific periodic trajectories (the particles follow periodic trajectories that exhibit locking into certain paths in the physical space).

Other possible approaches are based on changing the nature of the force driving particle motion (and eventually fluid flow) in the considered domain. 
Control of particle motion through static and rotating magnetic fields is being actively pursued by several groups (see, e.g., Zhou et al., ${ }^{22}$ and references therein). Similar techniques are based on electric fields (Behjatian and Esmaeeli ${ }^{23}$ ). More recently, in a range of particle sizes for which inertial forces are negligible, other investigators have tried to implement particle "activation" and control strategies based on the application of optical forces to light-reflecting nanoparticles. As an example, Delgado-Buscalioni et al., ${ }^{6}$ found that above a critical field intensity and concentration, the interplay between optical forces, thermal fluctuations and hydrodynamic pairing can lead particles (moving in aqueous solution under a nonconservative optical vortex lattice) to a spontaneous transition towards a kind of synchronised motion (in which they move along the diagonals of the lattice forming unidirectional currents).

However, there are many instances, where due to materials properties or other considerations of a practical nature, the use of such forces or combined strategies is not a viable option. In such cases, vibrational control has been considered as an attractive alternative. One might envisage using vibration to either producing a particle carrier flow with desired characteristics (Mialdun et al., ${ }^{24}$; Lyubimova et al., ${ }^{25}$; Lappa, ${ }^{26}$ ) or inducing particle segregation phenomena via the inter-particle hydrodynamic interactions. In the latter case related phenomena come under the heading of "mechanically forced" particle aggregation (see, e.g., Refs 27-32). Although here we do not embark in a detailed description of the related principles, the reader will easily realize that this category of separation methods can be applied only to concentrated dispersions (where the inter-particle hydrodynamic interactions are significant).

On the theoretical side, very recently, some new developments have appeared which deserve some attention as well. Such a new class of strategies, which seem to overcome the typical limitations of the method described above by extending the range of practical applicability of vibration-induced particle clustering to dilute systems, relies on the combined use of a time-periodic force field (acting on the ensemble of particles due to their different density with respect to the surrounding fluid medium) and the buoyant vibro-convective transport of solid phase produced by a carrier flow of thermal origin. Some recent contributions relating to this parallel line of inquiry are Refs 33-36. The reader may also be interested to know that the approach is conceptually similar to that developed by Ndukaife et al., ${ }^{37}$, the main difference being represented by the typical scale of our particles (for which inertia is significant) and the replacement of standard buoyancy flow (induced by steady gravity) with a time-varying acceleration.

Starting from the cases previously treated in the above-mentioned works, where the direction of imposed vibrations was assumed to be fixed in time (a given orientation in space), here we will consider the dynamics of a non-isothermal incompressible fluid with dispersed solid (inertial) particles in the case in which the cavity occupied by the fluid performs translational vibrations along a direction that is not constant in time as it undergoes a relatively slow and continuous rotation about a fixed axis. 


\section{Mathematical model}

Vibrations can be modelled as a unidirectional, harmonically oscillating, small-amplitude gravitational field (see, e.g., Monti et al., ${ }^{38,39}$ ):

$\underline{g}(t)=g_{\omega} \sin \left(\omega \mathrm{t}_{\mathrm{dim}}\right) \underline{\hat{n}}$

where $\mathrm{t}_{\mathrm{dim}}$ is the dimensional time and $g_{\omega}=\mathrm{b} \omega^{2}(\mathrm{~b}$ and $\omega$ being the amplitude and angular frequency of vibrations, respectively).

As reference geometric model we assume the classical cubic cavity already considered by Lappa $^{34,36}$. The related physical domain has characteristic size $L$ and, as shown in Fig. 1, it is delimited by a cooled wall located at $\mathrm{z}=-0.5$, a heated wall located at $\mathrm{z}=0.5$, while adiabatic conditions are applied to the remaining sides. We fix the value of the Prandtl number to $\operatorname{Pr}=8$, corresponding in nature to sodium nitrate $\left(\mathrm{NaNO}_{3}\right)$.

While steady gravity is not present in such a physical system (i.e. $\mathrm{Ra}=0$ ), we model the vibrations as periodic accelerations contained in the xy plane, forming a variable angle $\phi$ with the $\mathrm{x}$ axis:

$\phi=\Omega \mathrm{t}_{\mathrm{dim}}$

where $\Omega$ is a constant angular velocity satisfying the relationship.:

$O\left(10^{-2}\right) \leq \frac{\Omega}{\omega} \leq O(1)$

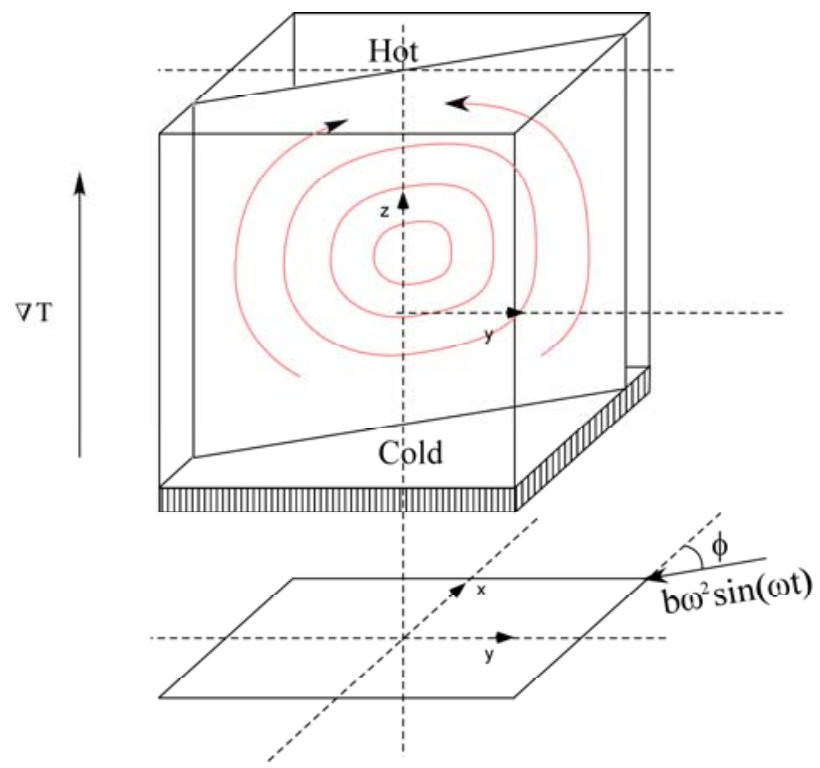

Figure 1: Cubic cavity with characteristic size $L$ shown, delimited by solid walls (one at $\mathrm{z}=-0.5$ cooled, the other at $\mathrm{z}=0.5$ heated, adiabatic conditions on the remaining sidewalls). The vibrations are contained in the xy plane and form an angle $\phi$ with the $\mathrm{x}$ axis that varies in time as $\phi=\Omega \mathrm{t}$, where $\Omega$ is a constant angular velocity. 
Such an acceleration field produces a fluctuating body force varying in time and space if density gradients are present. The resulting force field, not to be confused with that induced by the application of purely translational vibrations to a system rotating in space (our cavity does not rotate, which explains why we do not consider centrifugal and Coriolis effects) can be modelled through the addition of a dedicated source term at the right hand side of the balance equation for the fluid momentum.

Using the same conventional reference quantities of Refs 34 and 36 (i.e. defining the nondimensional time as $\mathrm{t}=\mathrm{t}_{\operatorname{dim}} L^{2} / \alpha$ and the reference velocity as $\alpha / \mathrm{L}$ where $\alpha$ is the thermal diffusivity), such equation together with the governing equations for mass and energy can be cast in non-dimensional form as

$\underline{\nabla} \cdot \underline{\mathrm{V}}=0$

$\frac{\partial \underline{V}}{\partial t}=-\underline{\nabla} p-\underline{\nabla} \cdot[\underline{V} \underline{V}]+\operatorname{Pr} \nabla^{2} \underline{V}+\operatorname{Pr} \frac{b \omega^{2} \beta_{T} \Delta T L^{3}}{v \alpha} T \sin \left(\frac{L^{2} \omega}{\alpha} t\right) \underline{\hat{n}}$

$\frac{\partial T}{\partial t}+\underline{\nabla} \cdot[\underline{V} T]=\nabla^{2} T$

where $\frac{b \omega^{2} \beta_{T} \Delta T L^{3}}{v \alpha}=R a_{\omega}$ is an analogue of the classical Rayleigh number, which emerges as a typical characteristic number in standard problems of thermogravitational convection when such problems are treated in the framework of the Boussinesq model (such approximation is used in the present work as well, $\beta_{\mathrm{T}}$ being the thermal expansion coefficient used to make the relationship between fluid density and temperature linear).

Thereby, the entire problem can be characterized conveniently in terms of five independent nondimensional parameters, namely, the well-known Prandtl number $(\operatorname{Pr}=v / \alpha$ where $v$ is the fluid kinematic viscosity), the buoyancy factor $(\beta)$, the nondimensional acceleration amplitude $(\gamma)$, the nondimensional angular frequency $(\varpi)$ and the nondimensional angular velocity $(\psi)$ with which the direction of vibrations rotates in space:

$\beta=\left(\beta_{T} \Delta T\right)$

$\gamma=\frac{b \omega^{2} L^{3}}{\alpha^{2}}$

$\varpi=\frac{\omega L^{2}}{\alpha}$ 
$\psi=\frac{\Omega L^{2}}{\alpha}$

Indicating the velocity components in the $\mathrm{x}, \mathrm{y}$ and $\mathrm{z}$ directions with $u, v$ and $w$, respectively, and projecting the governing equations along these directions yields in the container (fixed) frame:

$$
\begin{aligned}
& \frac{\partial u}{\partial t}=-\frac{\partial p}{\partial x}-(\underline{V} \cdot \underline{\nabla} \underline{V})_{u}+\operatorname{Pr}(\Delta \underline{V})_{u}+\gamma \beta T \sin (\varpi t) \cos \left(\varphi_{0}-\psi t\right) \\
& \frac{\partial}{\partial}=-\frac{\partial p}{\partial y}-(\underline{V} \cdot \underline{\nabla} \underline{V})_{v}+\operatorname{Pr}(\Delta \underline{V})_{v}+\gamma \beta T \sin (\varpi t) \sin \left(\varphi_{0}-\psi t\right) \\
& \frac{\partial w}{\partial}=-\frac{\partial p}{\partial z}-(\underline{V} \cdot \underline{\nabla} \underline{V})_{w}+\operatorname{Pr}(\Delta \underline{V})_{w}
\end{aligned}
$$

where $\underline{V}$ is the velocity, $\mathrm{p}$ is the pressure and

$$
\begin{aligned}
& (\underline{V} \cdot \underline{\nabla} \underline{V})_{u}=\left(u \frac{\partial u}{\partial x}+v \frac{\partial u}{\partial y}+w \frac{\partial u}{\partial z}\right) \\
& (\underline{V} \cdot \underline{\nabla} \underline{V})_{v}=\left(u \frac{\partial}{\partial x}+v \frac{\partial v}{\partial y}+w \frac{\partial v}{\partial z}\right) \\
& (\underline{V} \cdot \underline{\nabla} \underline{V})_{w}=\left(u \frac{\partial w}{\partial x}+v \frac{\partial w}{\partial y}+w \frac{\partial w}{\partial z}\right) \\
& (\Delta \underline{V})_{u}=\left(\frac{\partial^{2} u}{\partial x^{2}}+\frac{\partial^{2} u}{\partial y^{2}}+\frac{\partial^{2} u}{\partial z^{2}}\right) \\
& (\Delta \underline{V})_{v}=\left(\frac{\partial^{2} v}{\partial x^{2}}+\frac{\partial^{2} v}{\partial y^{2}}+\frac{\partial^{2} v}{\partial z^{2}}\right) \\
& (\Delta \underline{V})_{w}=\left(\frac{\partial^{2} w}{\partial x^{2}}+\frac{\partial^{2} w}{\partial y^{2}}+\frac{\partial^{2} w}{\partial z^{2}}\right)
\end{aligned}
$$

As in Refs 34 and 36, at the initial instant $(\mathrm{t}=0)$, a linear temperature distribution along the $\mathrm{z}$ axis is considered, i.e. $\mathrm{T}=\mathrm{z}+1 / 2$, while the fluid is assumed to be at rest (i.e. $\underline{V}=0$ everywhere). No-slip conditions are considered for all the solid walls.

Particles require a separate treatment. As explained in the introduction, since the nonzero mass and finite size of a particle can alter its motion locally, the motion of such a particle in the fluid will typically differ from that of an ideal tracer, which would simply follow the local velocity of the large-scale flow provided by the solution of eqs. (3-9). 
Accounting for these processes requires, in general, fast numerical methods able to resolve efficiently the considered multiscale system (which can include a relatively high number of particles, e.g. of $\mathrm{O}\left(10^{5}\right)$ in the present work). Historically, related computational strategies have been generally conceived to tackle some specific dynamical regimes and have been categorized accordingly (see, e.g., the Balboa Usabiaga et al., ${ }^{40}$ and references therein). Many of such methods display several advantages, which naturally make them potential candidates to be used as multiscale solvers. Nevertheless, some of them are not free of bottlenecks, which limit their practical applicability in many circumstances, unless the number of particles to be tracked is relatively small. Sifting through the available schemes to be potentially used attack the "multi-scale" problem considered here, in particular, the approach originally introduced by Maxey and Riley ${ }^{41}$ may be regarded as one of the most relevant and/or convenient due to its well-known flexibility and reduced computational cost and time (as implicit in a pointwise particle descriptions).

Using the same scalings already used to put the governing equations for fluid motion in nondimensional form, the additional Lagrangian equations required to track the motion of particles (the so-called Maxey and Riley equation) can be written as:

$$
\begin{aligned}
& \frac{d u_{\text {partcl }}}{d t}=\frac{1}{\xi+1 / 2}\left[-\frac{\operatorname{Pr}}{S t}\left(u_{\text {partcl }}-u\right)+\frac{3}{2} \frac{d u}{d t}+\frac{3}{2}(\underline{V} \cdot \underline{\nabla} \underline{V})_{u}\right]+\frac{\xi-1}{\xi+1 / 2} \gamma \sin (\varpi t) \cos \left(\varphi_{0}-\psi t\right) \\
& \frac{d v_{\text {partcl }}}{d t}=\frac{1}{\xi+1 / 2}\left[-\frac{\operatorname{Pr}}{S t}\left(v_{\text {partcl }}-v\right)+\frac{3}{2} \frac{d v}{d t}+\frac{3}{2}(\underline{V} \cdot \underline{\nabla} \underline{V})_{v}\right]+\frac{\xi-1}{\xi+1 / 2} \gamma \sin (\varpi t) \sin \left(\varphi_{0}-\psi t\right) \\
& \frac{d w_{\text {partcl }}}{d t}=\frac{1}{\xi+1 / 2}\left[-\frac{\operatorname{Pr}}{S t}\left(w_{\text {partcl }}-w\right)+\frac{3}{2} \frac{d w}{d t}+\frac{3}{2}(\underline{V} \cdot \underline{\nabla} \underline{V})_{w}\right]
\end{aligned}
$$

where $\underline{V}_{\text {part }}\left(\mathrm{u}_{\text {part }}, \mathrm{v}_{\text {part }}, \mathrm{w}_{\text {part }}\right)$ is the particle velocity. Moreover, $\xi$, is the ratio of the particle to the fluid density, and $S t$ is the Stokes number (defined a $S t=\tau v / \mathrm{L}^{2}$ where $\tau$ is the particle relaxation time, $\tau=2 \mathrm{R}^{2} / 9 \mathrm{v}$, and $\mathrm{R}$ is the particle radius, particles being assumed to be perfect spheres).

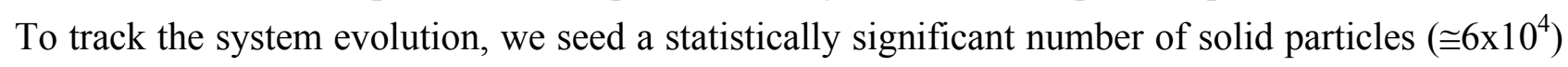
into the computational domain (see Fig. 2) assuming their initial distribution to be uniform (particles evenly spaced in the physical domain) and $\underline{V}_{\text {part }}=0$ as initial velocity. 


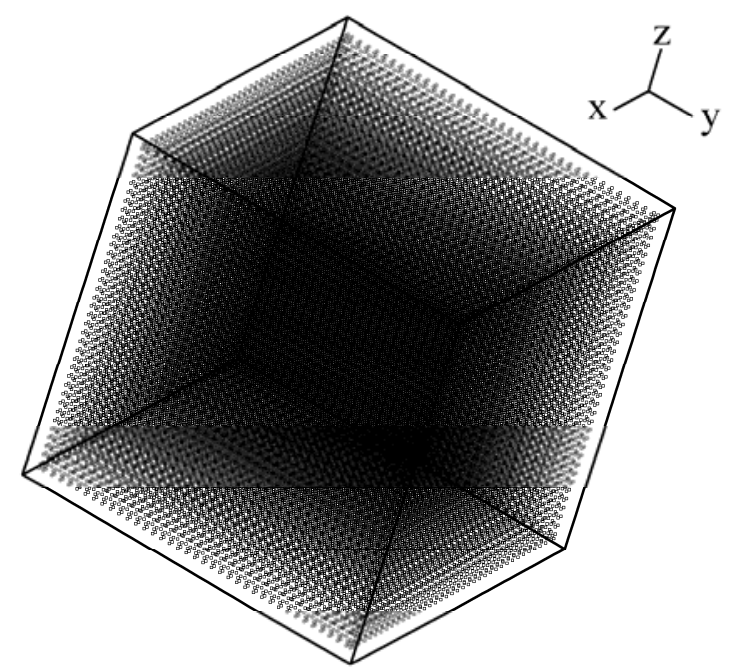

Figure 2: Initial distribution of particles

For the sake of conciseness and to avoid redundancy with respect to our earlier works (see, e.g., Kuhlmann et al., ${ }^{42}$ for the rationale underlying the so-called one-way approach applicable to dilute systems), as a concluding remark for this section we limit ourselves to mentioning that the mass, momentum and energy balance equations for the fluid phase have been solved in the framework of a classical projection method with standard staggered arrangement of the variables on the underlying structured Cartesian grid and central-differencing schemes in space (second order accurate, see Lappa ${ }^{34,43}$ for additional details, which are not duplicated here to save space). Coupling of such a strategy with particle tracking, i.e. eq. (10), was already implemented in Ref 33. For an assessment of the impact of the interpolation schemes required to reconstruct the fluid velocity at the generic particle position and the mesh resolution on the accuracy of the resulting numerical method, the reader may consult Refs 35 and 44.

\section{Results and discussion}

The properties of the considered solid-liquid system are summarized in Table I.

TABLE I: Liquid-solid system properties

\begin{tabular}{ll}
\hline \hline Fluid $\left(\mathrm{NaNO}_{3}\right)$ density $\rho, \mathrm{kg} \mathrm{m}^{-3}$ & 1904 \\
Kinematic viscosity $\nu, \mathrm{m}^{2} \mathrm{~s}^{-1}$ & $1.27 \times 10^{-6}$ \\
Thermal diffusivity $\alpha, \mathrm{m}^{2} \mathrm{~s}^{-1}$ & $1.58 \times 10^{-7}$ \\
Thermal expansion coefficient $\beta_{\mathrm{T}}, \mathrm{K}^{-1}$ & $1.25 \times 10^{-3}$ \\
Particle/liquid density ratio $\xi$ & 1.85 \\
\hline \hline
\end{tabular}


The following values of characteristic nondimensional parameters are considered: $\xi=1.85, \eta=10^{-4}$, where $\eta=(\xi-1) \operatorname{St} / \operatorname{Pr}$. Moreover, $\varpi=10^{3}, \gamma=1.5 \times 10^{6}$ which taken together give $\operatorname{Ra}_{\omega}=1.58 \times 10^{4}$. The corresponding value of the so-called Gershuni number reads Gs $=\frac{1}{2 \operatorname{Pr}}\left(\frac{\beta \gamma}{\varpi}\right)^{2}=10^{3}$. This guarantees that time-averaged convective effects of thermovibrational nature are very small with respect to the oscillatory component of fluid velocity and that the departure of the time-averaged temperature field from the purely linear and diffusive conditions is almost negligible ${ }^{34,36}$. This also means that neither boundary layers of thermal origin nor steep gradients of velocity are present in the considered system ${ }^{35}$. The absence of such regions, in turn, supports the main outcomes of our mesh assessment study (in which a mesh $42 \times 42 \times 42$ was found to be sufficient to achieve grid independence).

As discussed in Sect.2, the characteristic nondimensional angular velocity $\psi$ defined by eq. (6d) is allowed to span a relatively wide range, i.e.

$10 \leq \psi \leq 2 \times 10^{3}$

which, in terms of the additional parameter

$\sigma=\psi / \varpi$

reads

$10^{-2} \leq \sigma \leq 2$

Prior to embarking onto a detailed description of the present findings, in the following we shortly review past results obtained using the same framework ${ }^{34}$. Indeed, for the convenience of the reader, it is helpful to start the discussion from the simplified situation where the direction of vibrations is fixed in time. Related results, summarized in Fig. 3 and 4, provide evidence for the ability of vibrations to promote irreversible pattern formation phenomena driven by particles in nonisothermal fluids even in situations for which particle-particle hydrodynamic interactions are negligible (dilute dispersions for which the Lagrangian tracking equation introduced in Sect. 2 is valid). As shown in these figures, well-defined particulate structures are formed over "long" temporal scales, i.e. times much larger than the period of the external forcing.

For fixed $\phi$, the relative direction of applied vibrations with respect to the imposed temperature gradient and the axes of symmetry of the considered geometrical configuration can influence significantly the morphology of the particle attracting regions in the physical space, these being closed (a kind of polyhedrons) or non-compact (tubular surfaces) depending on which of the intrinsic reflection symmetries of the vibrated system are preserved or violated. 


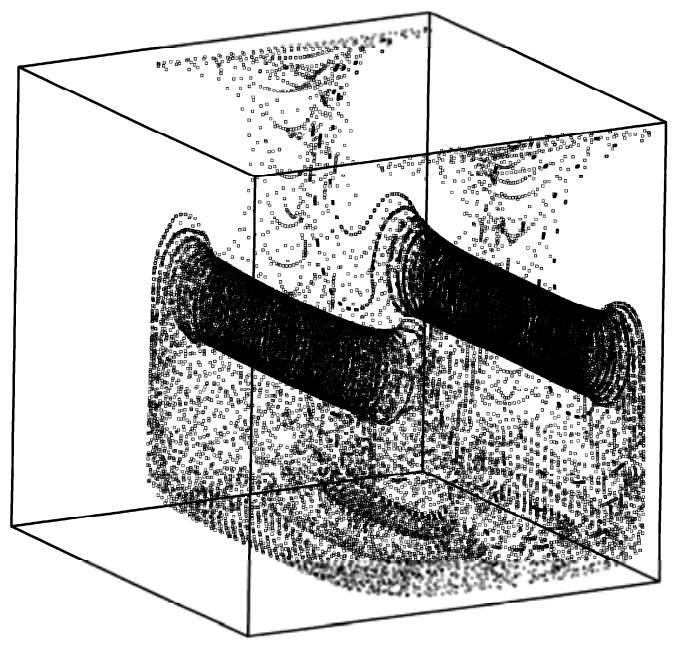

a)

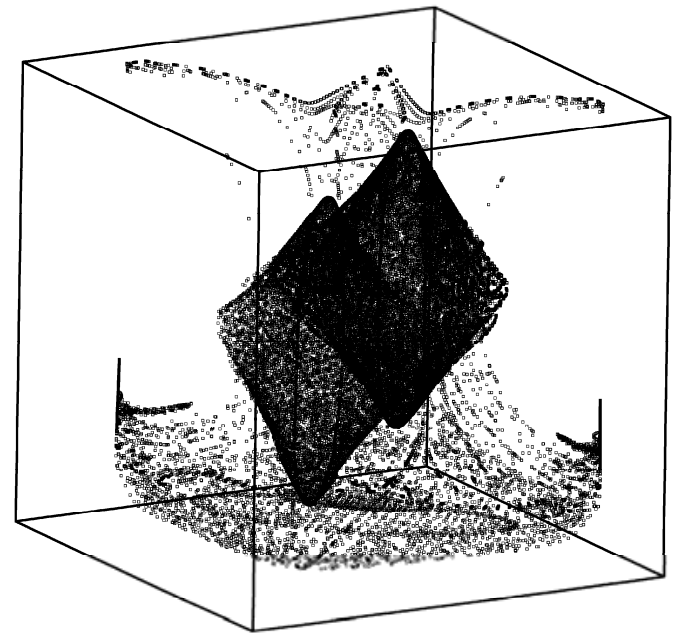

b)

Figure 3: Particle aggregates ( $3 \mathrm{D}$ view, $\mathrm{z}$ axis direction from top to bottom, $\mathrm{t}=0.7$ ) for $\varpi=1 \times 10^{3}$, $\psi=0, \gamma=1.5 \times 10^{6}, \mathrm{Ra}_{\omega}=1.58 \times 10^{4}, \xi=1.85$, and $\eta=1 \times 10^{-4}$ : a) vibrations along the $\mathrm{x}$ axis, $\phi=0, \mathrm{~b}$ ) vibrations along the $\mathrm{x}=\mathrm{y}$ direction, $\phi=\pi / 4$.

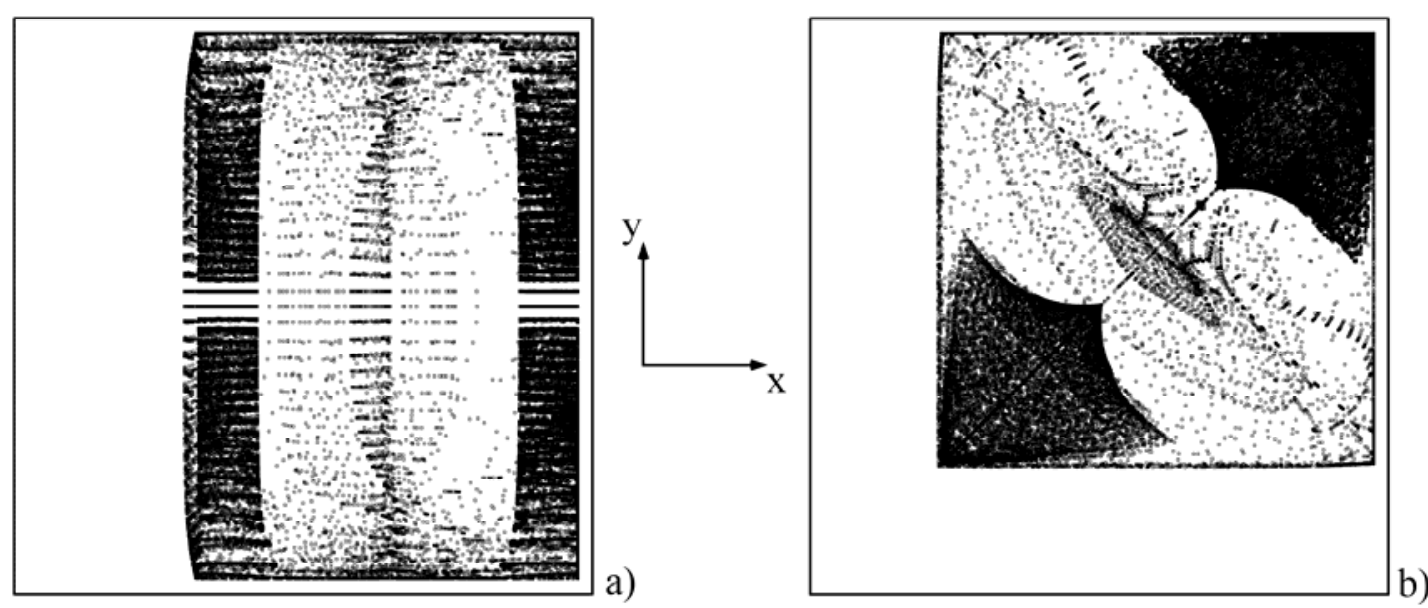

Figure 4: Particle aggregates (top view) for the same conditions of Fig. 3: a) vibrations along the $x$ axis, $\phi=0, \mathrm{~b}$ ) vibrations along the $\mathrm{x}=\mathrm{y}$ direction, $\phi=\pi / 4$.

As shown by Lappa ${ }^{34}$, regardless of the specific shape taken by the particle attracting regions, such a long-range translational ordering is produced by the delicate interplay between convective effects (of thermovibrational nature) and the (inertial) response of each isolated particle to the timeperiodic forcing.

This mechanism is still operative for the conditions considered in the present work as witnessed by the sequence of snapshots summarised in Figs. 5-6 and 7-8 for $\psi=10$ and $\psi=100$, respectively. As evident in such sequences, starting from an initially uniform distribution of particles, the application of vibrations with direction continuously changing in time forces particles to demix from the fluid and accumulate in certain sub-regions of the container. 


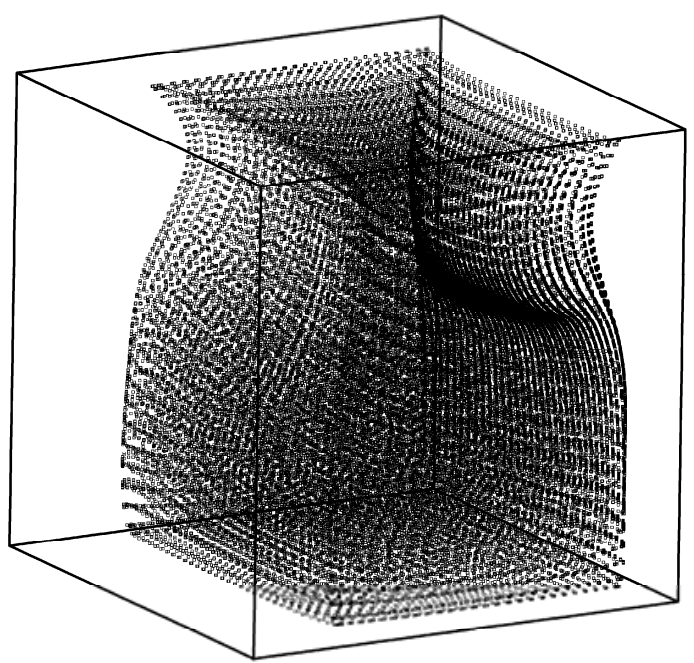

a)

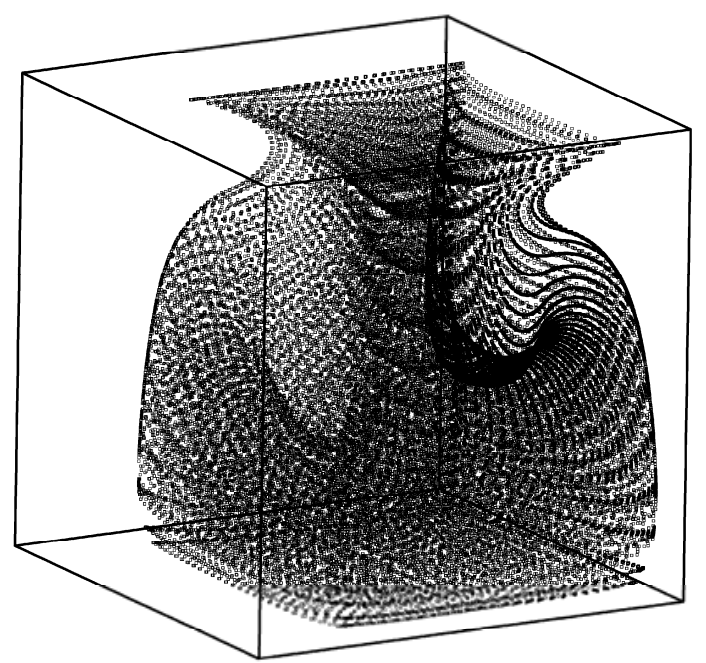

b)

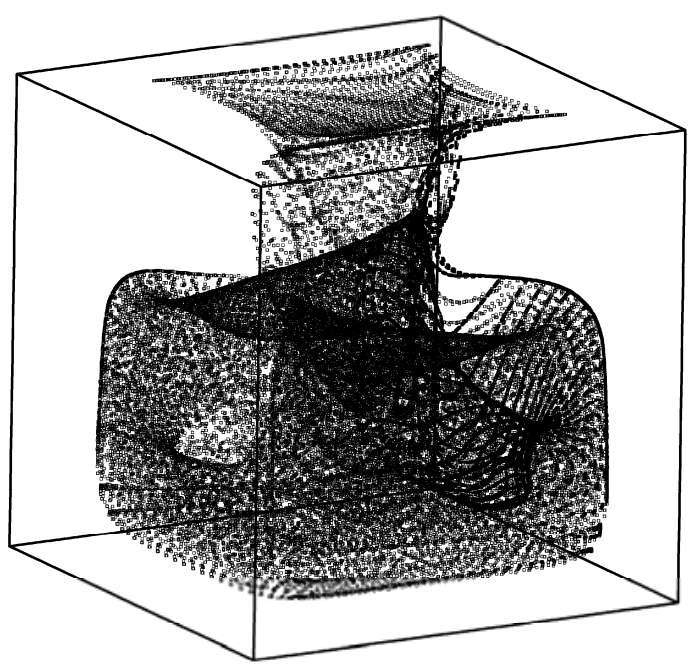

c)

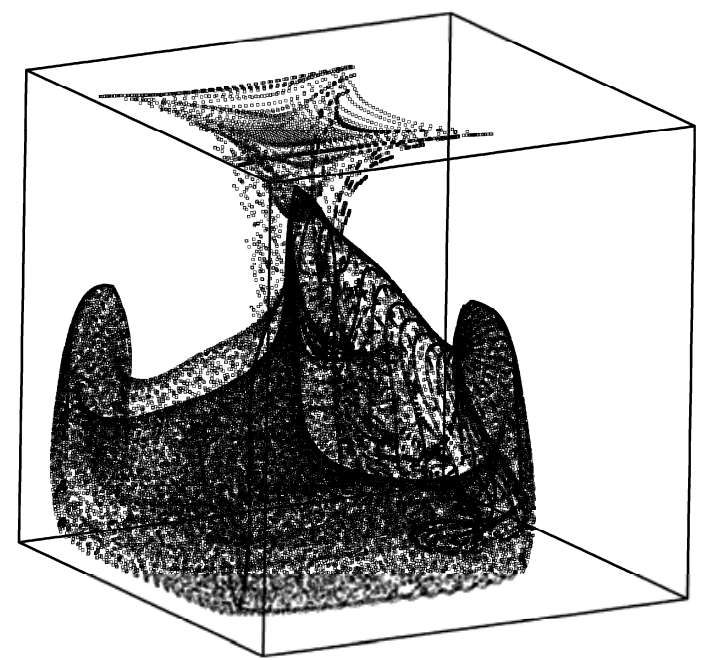

d)

Figure 5: Particle aggregates as a function of time for $\psi=10$ : a) $\left.t=5.5 \times 10^{-2}, b\right) t=1.1 \times 10^{-1}$, c) $\left.\mathrm{t}=2.2 \times 10^{-1}, \mathrm{~d}\right) \mathrm{t}=3.3 \times 10^{-1}$. 

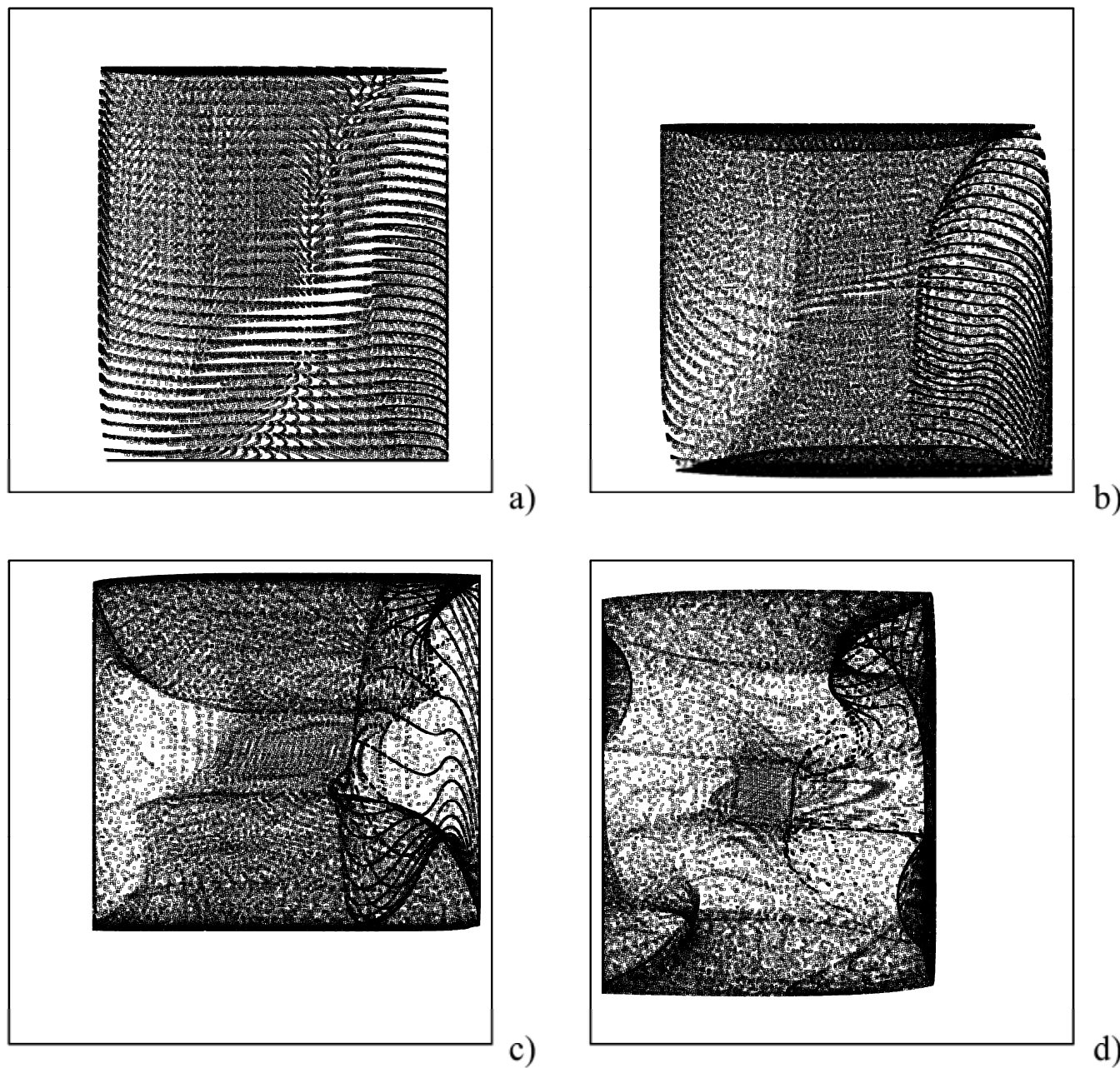

Figure 6: Top view of particle aggregates as a function of time for the same conditions considered in Fig. 5: a) $\left.\mathrm{t}=5.5 \times 10^{-2}, \mathrm{~b}\right) \mathrm{t}=1.1 \times 10^{-1}$, c) $\mathrm{t}=2.2 \times 10^{-1}$, d) $\mathrm{t}=3.3 \times 10^{-1}$. 


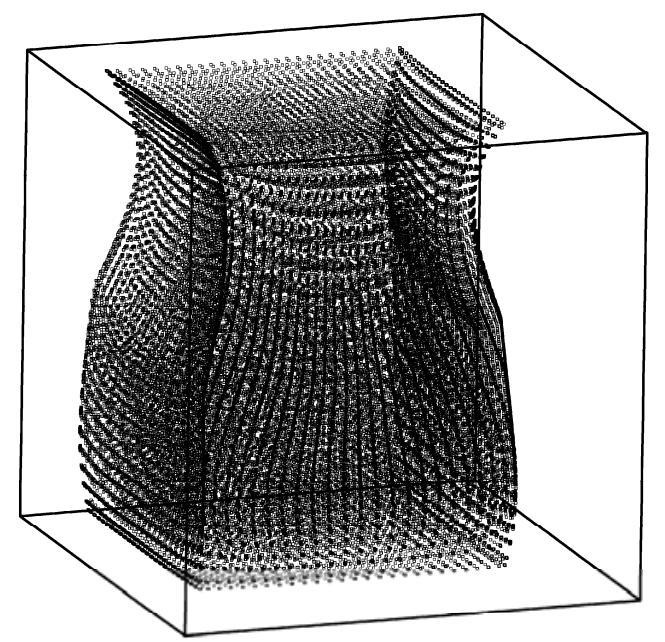

a)

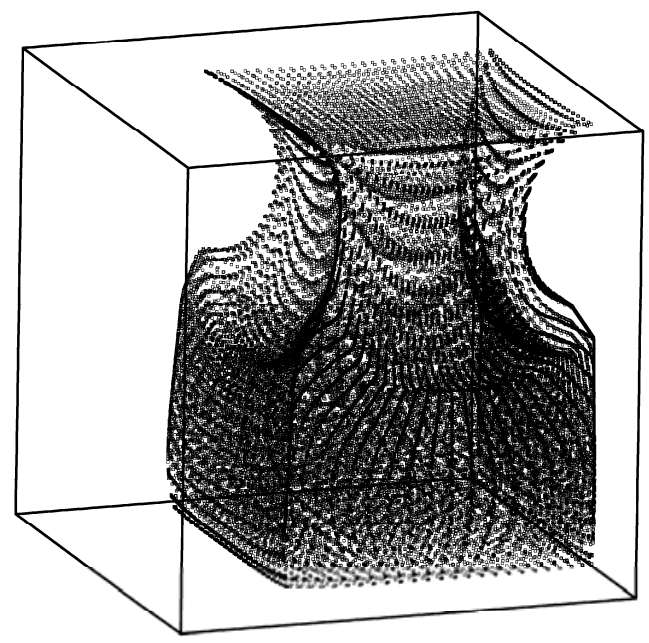

b)

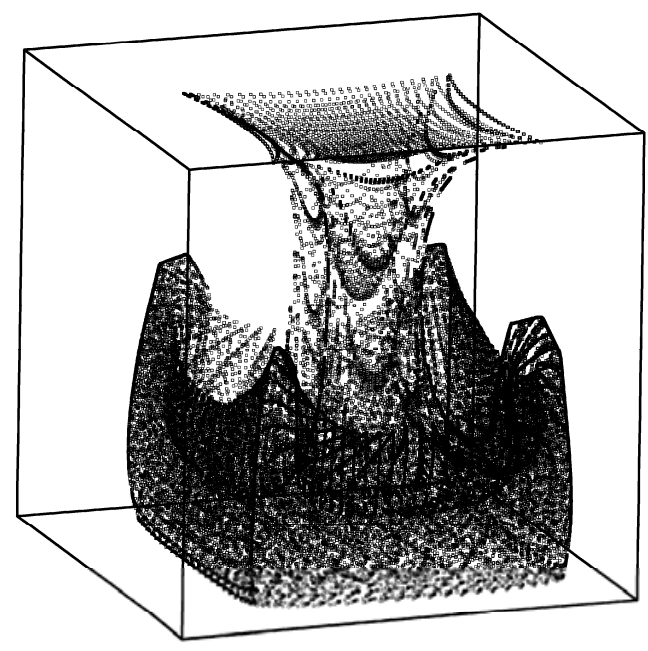

c)

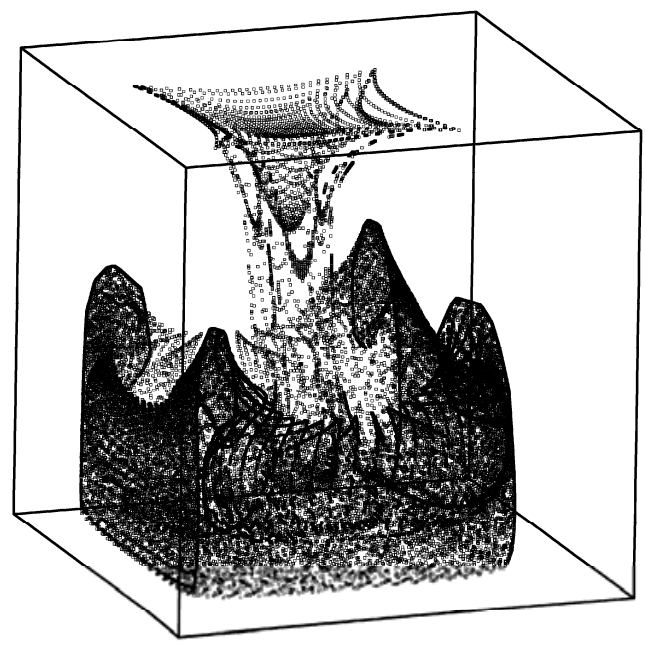

d)

Figure 7: Particle aggregates as a function of time for $\psi=1 \times 10^{2}:$ a) $\left.t=5.5 \times 10^{-2}, b\right) t=1.1 \times 10^{-1}$, c) $\left.\mathrm{t}=2.2 \times 10^{-1}, \mathrm{~d}\right) \mathrm{t}=3.3 \times 10^{-1}$. 

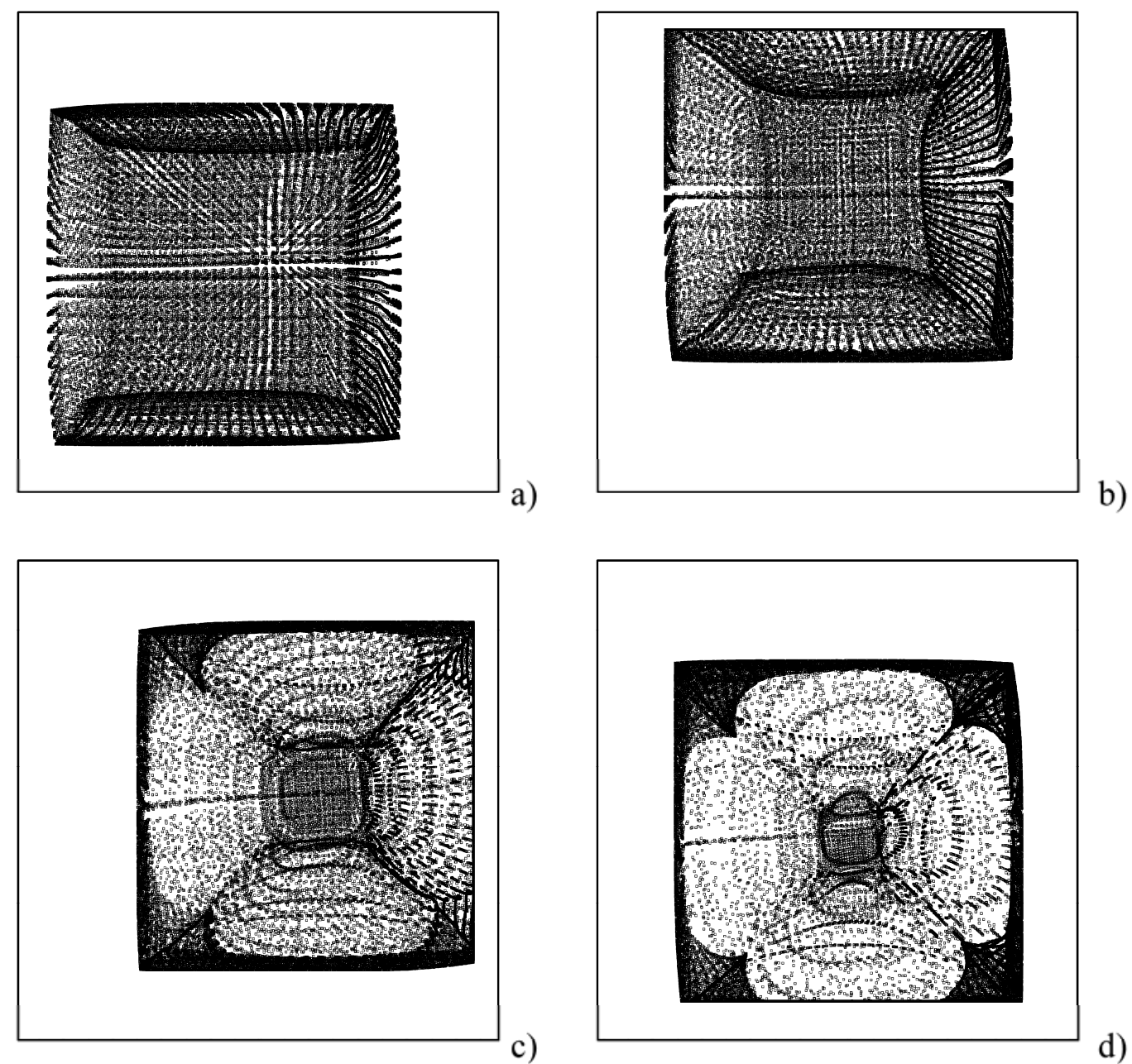

d)

Figure 8: Top view of particle aggregates as a function of time for the same conditions considered in Fig. 7: a) $\mathrm{t}=5.5 \times 10^{-2}$, b) $\left.\mathrm{t}=1.1 \times 10^{-1}, \mathrm{c}\right) \mathrm{t}=2.2 \times 10^{-1}$, d) $\mathrm{t}=3.3 \times 10^{-1}$.

Some of the general principles already identified by Lappa ${ }^{34}$ are still valid. Indeed, some precise spatial properties can be identified in the emerging structures. As seen from above (Figs. 6 and 8), the pattern displays a kind of central symmetry similar to that already observed in other phenomena involving particles dispersed in a fluid enclosed in a box (such as those produced by the propagation through a dusty gas of initially spherical shock waves originating from the centre of square containers ${ }^{10}$ ).

For the present conditions, this emerging property has a completely different nature as it can be ascribed to time-mediated nonlinear effects produced by the rotation of the vibration axis. On average, in fact, the cubic enclosure undergoing vibrations along a direction that rotates with a constant angular velocity about the $\mathrm{z}$ direction may be seen as a system possessing both the M2 and M4 diagonal-reflection properties depicted in Fig. 9. 


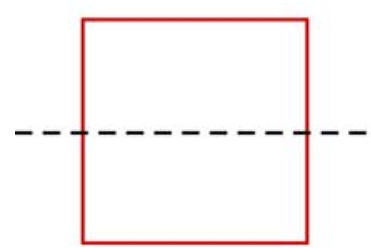

M1 reflection

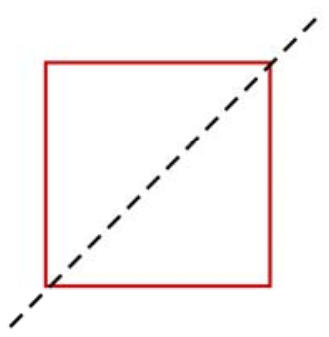

M2 reflection

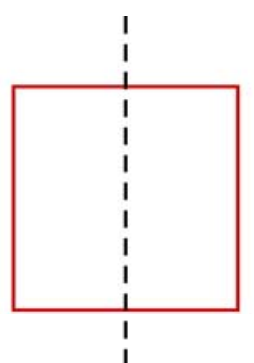

M3 reflection

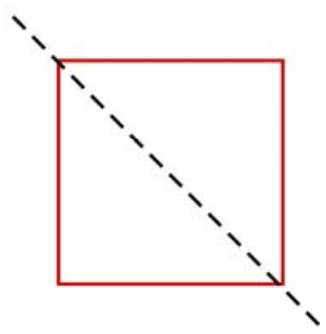

M4 reflection

Figure 9: The symmetry group of a square (reflection symmetries).

On the one hand, this argument may be used to justify the increased symmetry displayed by the particle pattern with respect to those revealed by earlier studies (where the symmetry of particle attractors was limited to the M1 (M3) or M2 (M4) reflections). On the other hand, it may be also used to explain the specific shape taken by the particle aggregates (polyhedron-like structures are formed in the corners, which resemble those emerging for the case of systems obeying the M2 or M4 symmetry, as illustrated in Fig. 3b).

A complete characterisation of these phenomena, however, also requires proper consideration of temporal effects. Indeed, comparison of Figures 6 and 8 clearly indicates that while for $\psi=10^{2}$ the pattern is allowed to reach a stationary state in terms of morphology and topology (the aforementioned recognizable structure with four polyhedrons located in the system corners), the attainment of such a condition is not possible for $\psi=10$ (which confirms that, as expected, the temporal scales of the considered forcing do play a role in determining the system response).

Adequate assessment of such trends requires proper "separation" of the different involved time scales. A first step along these lines is represented by consideration of the "trivial" condition $\psi=0$.

For this condition and the same value of the vibration frequency assumed in the present work, it is known $^{34}$ that once the recognizable typical particle structure is formed, a further increase of time, produces no variation in the morphological and topological properties of the pattern. Under the effect of vibrations the pattern oscillates back and forth along the vibration axis as a whole (giving the illusion of a solid unit) at the vibration frequency. This implies that in the reference frame attached to the container (shown in Fig. 1) the theoretical possibility exists to express the coordinates of each particle as the sum of a time-averaged steady component and a time-dependent (fluctuating) contribution. Indicating by $\underline{r}$ the position of the generic particle, the above principle may be formalized as:

$\underline{r}=\underline{\bar{r}}+\underline{r}^{\prime}(\varpi, t)$

where 
$\bar{r}=\frac{\varpi}{2 \pi} \int_{0}^{2 \pi / \pi} \underline{r} d t$

$\underline{r}^{\prime}=\underline{r}-\underline{\bar{r}}$

Given the above arguments about the property of the structures formed by particles to behave as they were a solid unit, it becomes evident that in the case $\psi=0$ consideration of the particle position in a coordinate system moving in space with the particles (hereafter referred to as starred "**" reference frame) would lead to $\underline{r}^{*},=0$. Such a reference system may be imaged to have its origin in the particle-structure barycentre. The position of such a point can be defined using the following general relationship:

$\underline{r}_{G}=\frac{1}{N_{\text {partcl }}} \sum_{i=1}^{N_{\text {partcl }}} \underline{r}_{i}$

Following the same concepts already used above, the corresponding time-averaged position (with respect to the period of vibrations $2 \pi / \varpi$ ) can be defined as

$\overline{\underline{r}}_{G}=\frac{\varpi}{2 \pi} \int_{0}^{2 \pi / \varpi} \underline{r}_{G} d t$

Accordingly:

$\underline{r}^{*}=\underline{\bar{r}}-\underline{r}_{G}$

Obviously, the theoretical possibility to split the local position of each particle into a time-averaged steady component plus an oscillating part in the standard reference frame (attached to the container) is retained in the case of rotating vibration axis $(\psi \neq 0)$. However, one should keep in mind that for such a situation, the oscillatory part of $\underline{r}$ will be made up of at least three contributions, one related to the rhythmic displacement induced by vibrations along the instantaneous direction of the vibration axis, another related to the rotation of such an axis, plus an additional contribution due to the nonlinear interaction of the $\varpi$ and $\psi$ oscillatory modes, namely:

$\underline{r}^{\prime}=\underline{r}_{1}(\varpi, t)+\underline{r}_{2}(\psi, t)+\underline{r}_{3}(\varpi, \psi, t)$

Most interestingly, the numerical results give evidence that this third contribution does not disappear in the starred coordinate system vibrating with respect to the fixed one at the frequency $\varpi$ and rotating with velocity $\psi$. In other words, in the moving reference frame, eq. (17) would read: 


$$
\underline{r}^{\prime^{*}}=\underline{r}_{3}^{*}(\varpi, \psi, t) \neq 0
$$
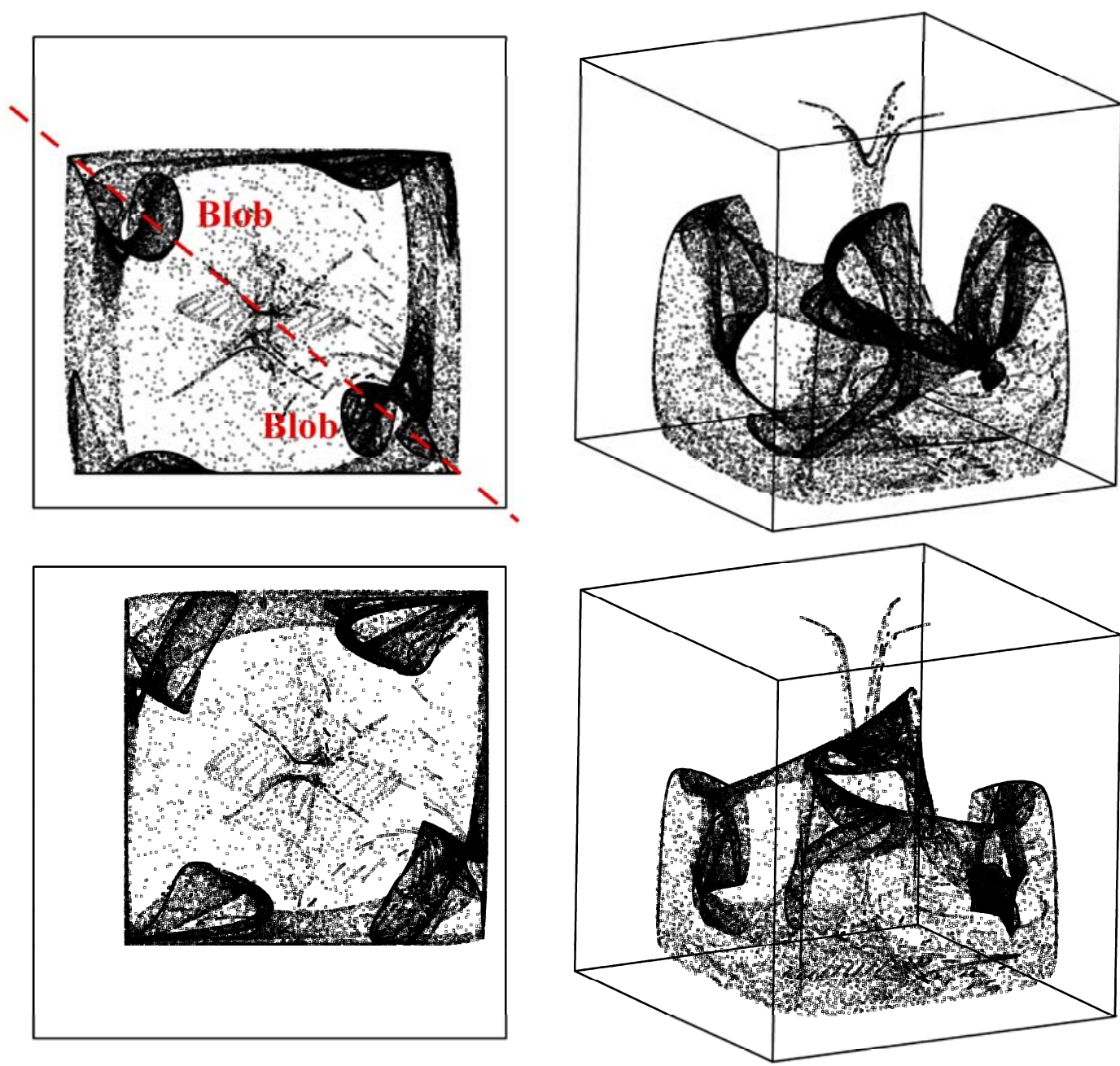

a)
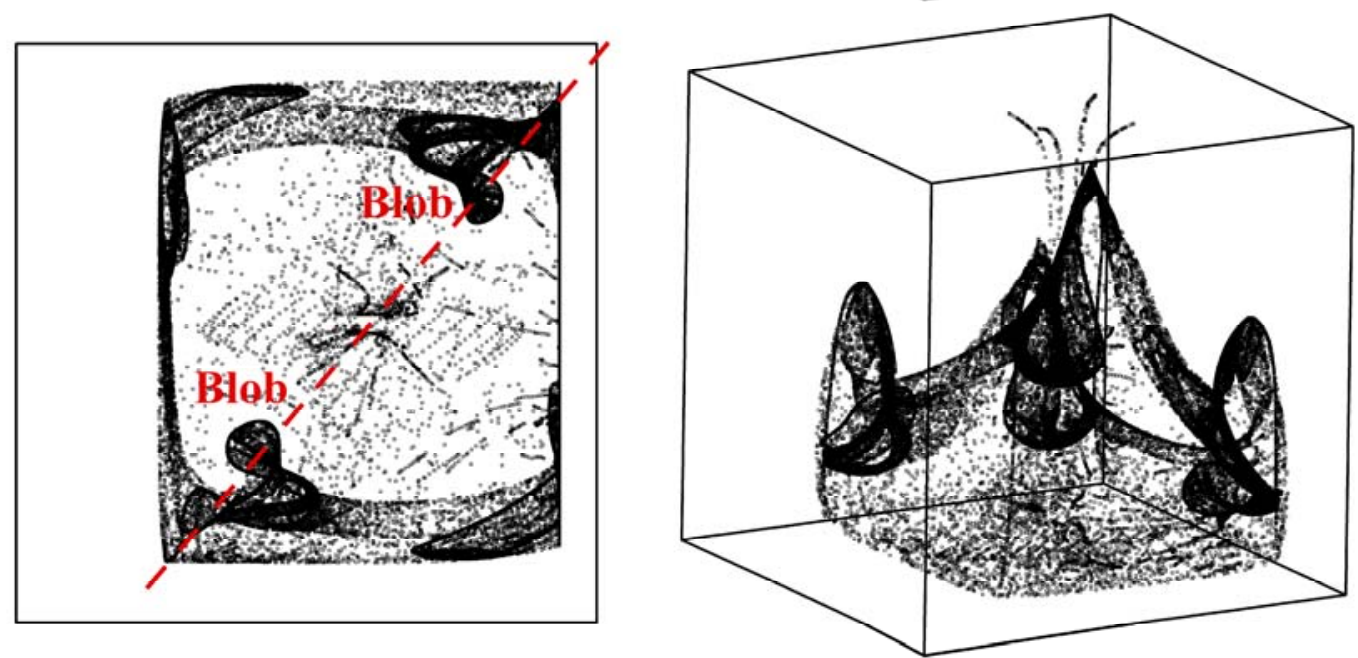

b)

c)

Figure 10: Top view (left) and side view (right) of particle aggregates as a function of time for $\psi=10$ during $1 / 4$ of the vibration axis rotation period $(\pi \psi / 2), \mathrm{t}_{0}=0.79$, time shift between two consecutive snapshots $\Delta \tau=6.28 \times 10^{-2}$. 
The numerical simulations also indicate that different situations (regimes) are established depending on the effective value of the parameter $\sigma=\psi / \varpi$.

For $\sigma=10^{-2}$, as clearly witnessed by the significant changes in shape (as time passes) undergone by the pattern visible in Fig. 6, there is a first regime characterized by a relatively large oscillatory component $\left|\underline{r}_{3}^{*}\right|$. In this case the asymptotic formation of a stationary pattern is prevented by the $\left|\underline{r}_{3}^{*}\right|$ contribution.
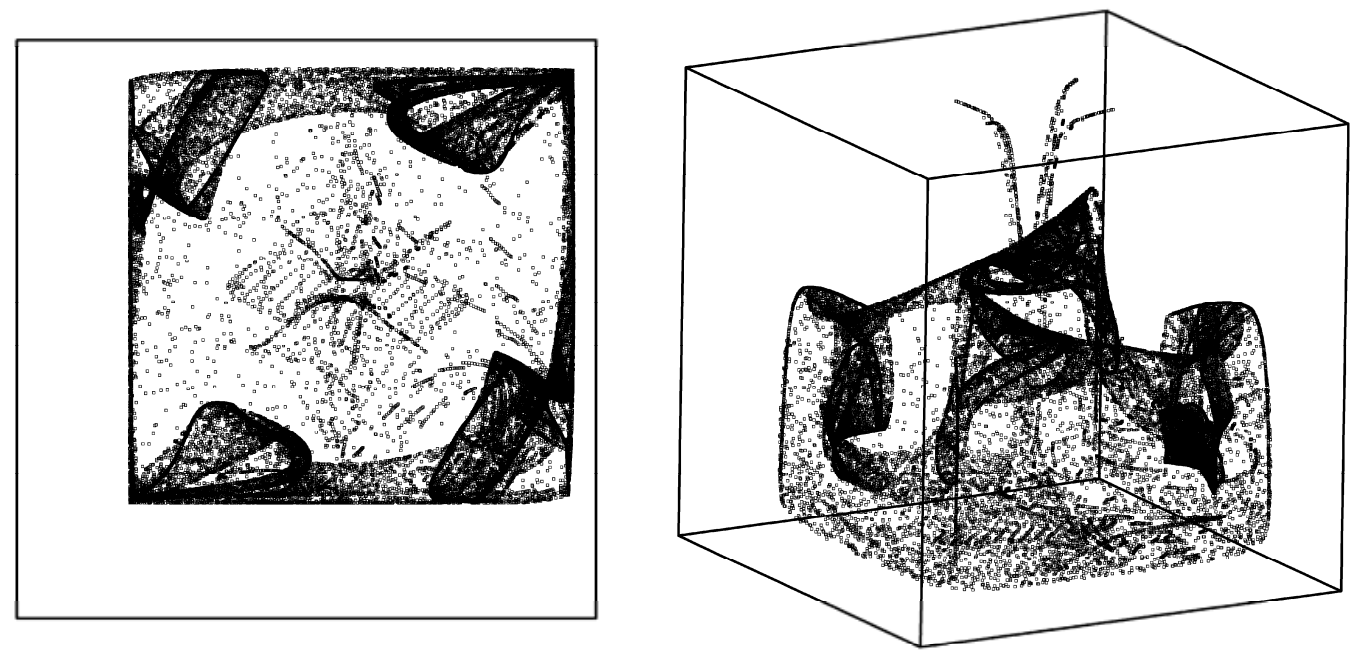

a)
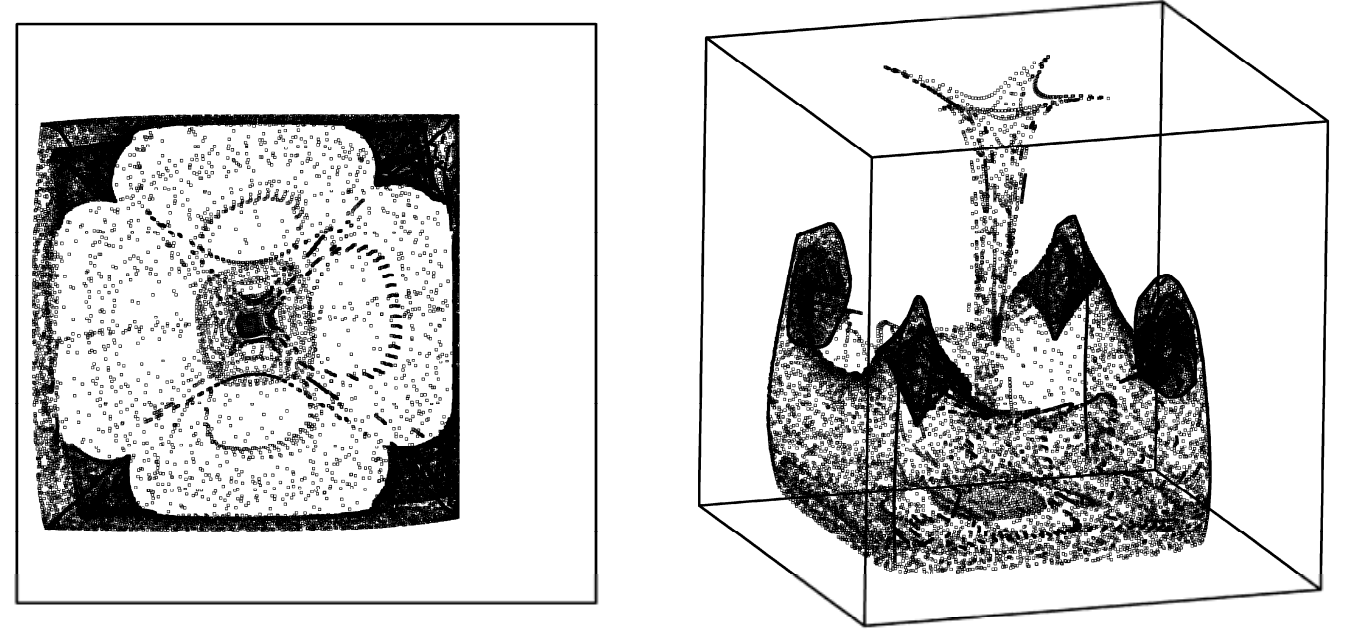

b)
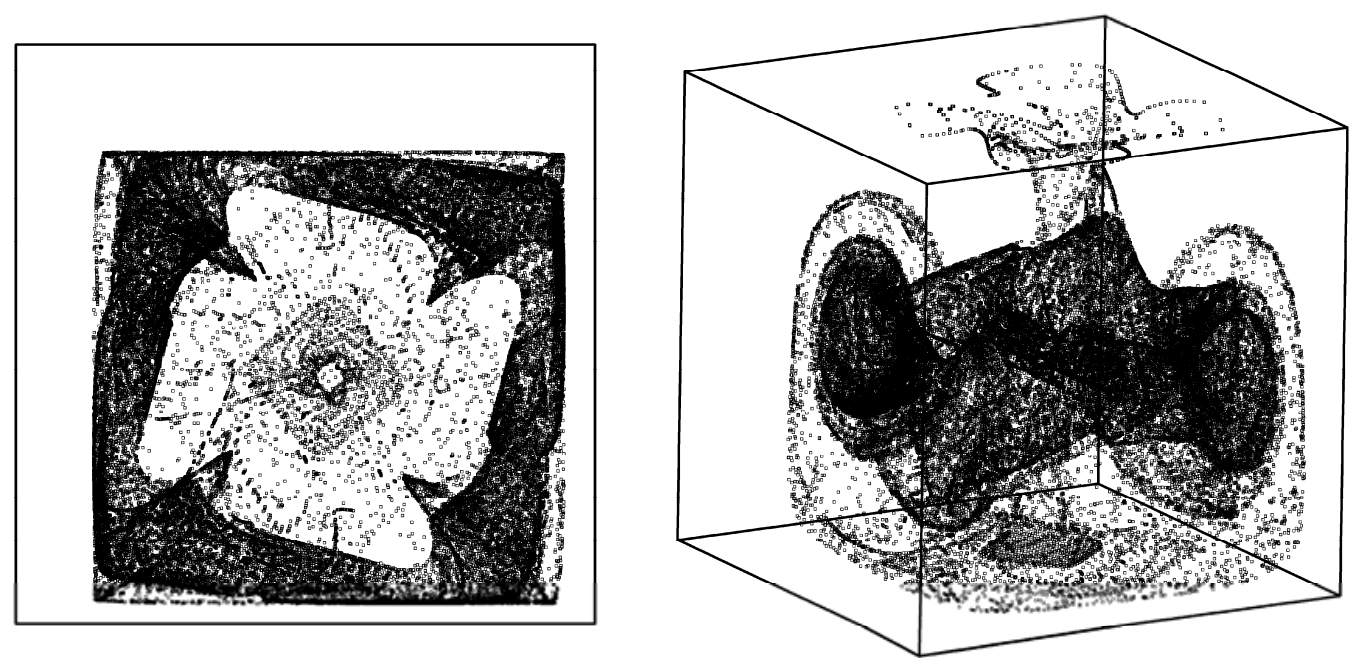

c)

Figure 11: Particle aggregates as a function of $\psi$. a) $\left.\psi=10, \mathrm{t}=8.5 \times 10^{-1}, \mathrm{~b}\right) \psi=100, \mathrm{t}=5.9 \times 10^{-1}$, c) $\psi=2000, \mathrm{t}=1$. 1 . 
As shown in Fig. 10, this contribution gives rise to an oscillatory pattern with period $\pi / \psi$, displaying (in the view from above) the periodic replacement of a structure with two localised regions of increased particle density (referred to as "blobs" in Fig. 10), initially aligned along the $x$ $=-\mathrm{y}$ direction (at $\mathrm{t}=\mathrm{to}$ ), with a structure in which the two blobs are aligned along the $\mathrm{x}=\mathrm{y}$ direction (at $\mathrm{t}=\mathrm{to}+1 / 2 \pi / \psi$ ).

By contrast, for $\sigma=10^{-1}$, the oscillatory contributions are relatively small with respect to the steady ones induced by time-average effects. Indeed, in such a case, in the view of the system from above the blobs appear to be steadily located in the corners and correspond to the formation of four recognizable particle accumulation structures having the morphology of polyhedrons (Fig. 11b, left and right views, respectively).
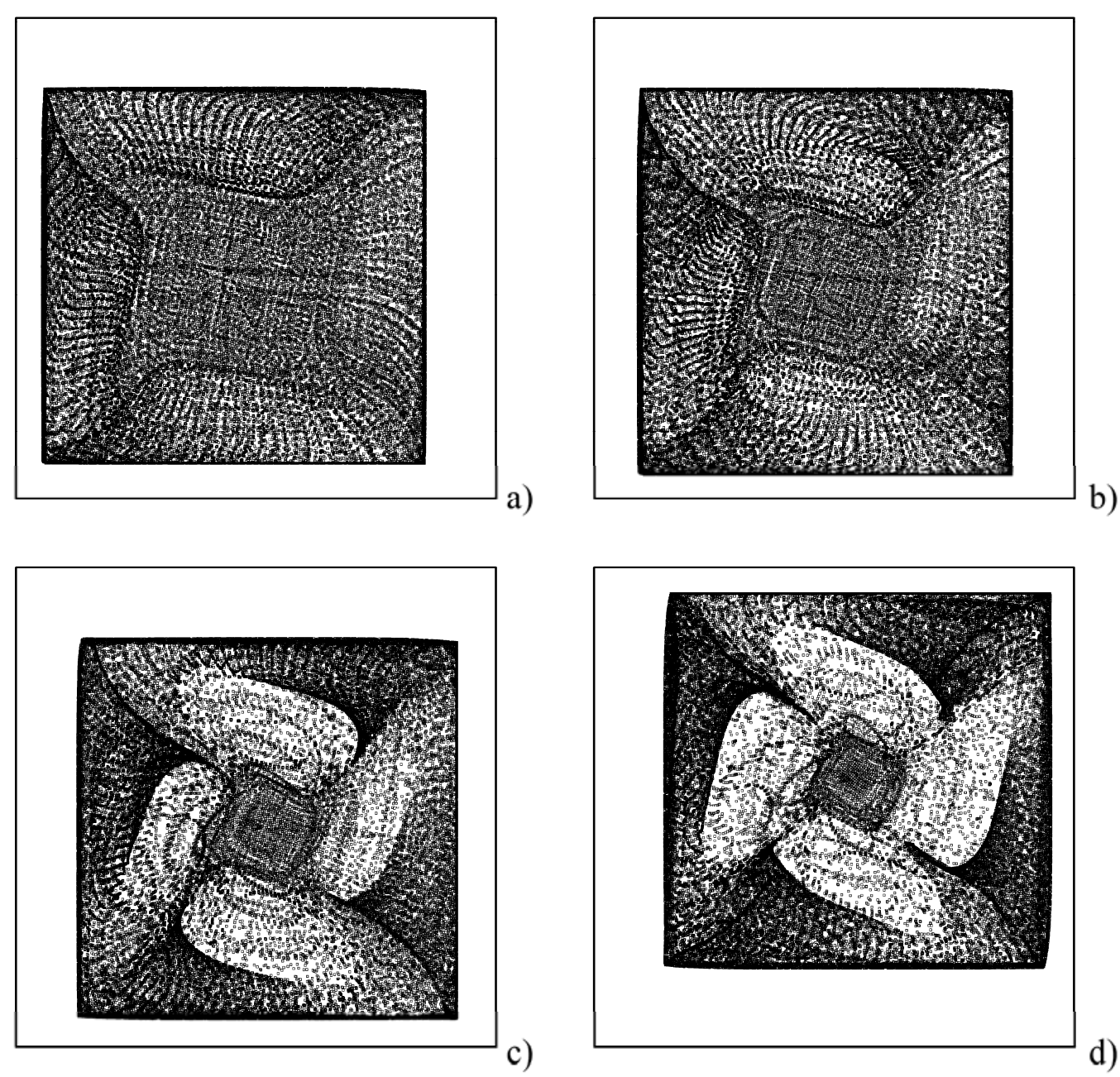

Figure 12: Top view of particle aggregates as a function of time for $\left.\psi=2 \times 10^{3}: a\right) t=2.417 \times 10^{-1}$, b) $\mathrm{t}=3.3 \times 10^{-1}$, c) $\mathrm{t}=5.06 \times 10^{-1}$, d) $\mathrm{t}=6.83 \times 10^{-1}$.

Most interestingly, a further increase in the angular velocity of the vibration axis $(\sigma=2)$, produces a state, which, albeit stationary in terms of spatio-temporal behaviour (no qualitative changes in time), is no longer featured by the centre-symmetry property seen for $\sigma=\mathrm{O}\left(10^{-1}\right)$ (Fig. 11c, the reader 
being also referred to Fig. 12 for the related initial stages of evolution). The structure formed by particles takes the shape of a four-leaf clover with asymmetric lobes (a sort of a four-blade windmill). Interestingly, this may indicate that the system has entered a new regime characterised by the rupture of symmetry. This conjecture is also supported by the existence of a resonant state for $\sigma=1$ where no recognizable particle structures are formed (not shown). Such a value of $\sigma$ may be regarded as a threshold separating states with centre-symmetry with states in which such a symmetry is broken.

These results are complemented by those shown in Fig. 13. This final figure confirms that for a fixed $\varpi$, in general, $\left|\underline{r}_{3}^{*}\right|$ behaves as a weakly decreasing function of $\sigma$. More precisely, we have reported in this figure the position of the particle-structure barycentre with respect to the standard reference frame as a function of time.

The amplitude of the oscillations reported in these figures may be regarded as a spatial average (via eq. (14)) of the module of the quantity $\underline{r}$ ' defined by eq. (17). It can be seen that higher values of $\psi$ lead to a decrease in the signal amplitude. As $\underline{r}_{1}$ does not depend on $\psi$, such a decrease must be ascribed to the shrinkage experienced (for increasing values of the angular velocity) by the other two components, i.e. $\underline{r}_{2}$ and $\underline{r}_{3}$ (only the latter contributing to time-dependent effects in the starred frame of reference).

Among other things, the right views of these figures and the corresponding red lines in the left views also give evidence of a weak departure of the time-averaged position of the barycentre from the position that it should ideally maintain in the absence of non-linear effects (corresponding to the point $\mathrm{x}=0, \mathrm{y}=0$ ). This is due to the fact that, though small (because of the small value of the Gershuni number), convective time-averaged effects in the fluid are not completely negligible (because of the finite, i.e. non-zero, value of this number: $\mathrm{Gs}=10^{3}$ for the present conditions).

\section{Conclusions}

The surprising phenomenon by which small particles added as tracers to a vibration-induced thermal buoyancy flow do not stay uniformly suspended but rather assemble in well-defined threedimensional objects or surfaces has been further explored by considering a pulso-rotating forcing (vibrations axis rotating about the direction of the imposed temperature gradient). Different scenarios have been identified according to the relationship between the two main temporal scales involved in the considered category of phenomena (namely, the period of vibrations and the period of rotation of the related axis).

For a period of rotation much larger than the vibrations period $\left(\sigma=10^{-2}\right)$, a first regime exists where the formation of an asymptotic pattern with stationary morphological and topological properties is prevented. The pattern alternates as time progresses between two limit configurations displaying recognizable symmetry properties with respect to the $\mathrm{x}=\mathrm{y}$ or $\mathrm{x}=-\mathrm{y}$ direction. In particular, these two limit states, resembling the patterns which would be obtained in the case of non-rotating axis for 
$\phi=\pi / 4$ and $\phi=-\pi / 4$, are attained when the system (cubic cavity + imposed vibrations axis) periodically satisfies the M2 or M4 reflection symmetry.
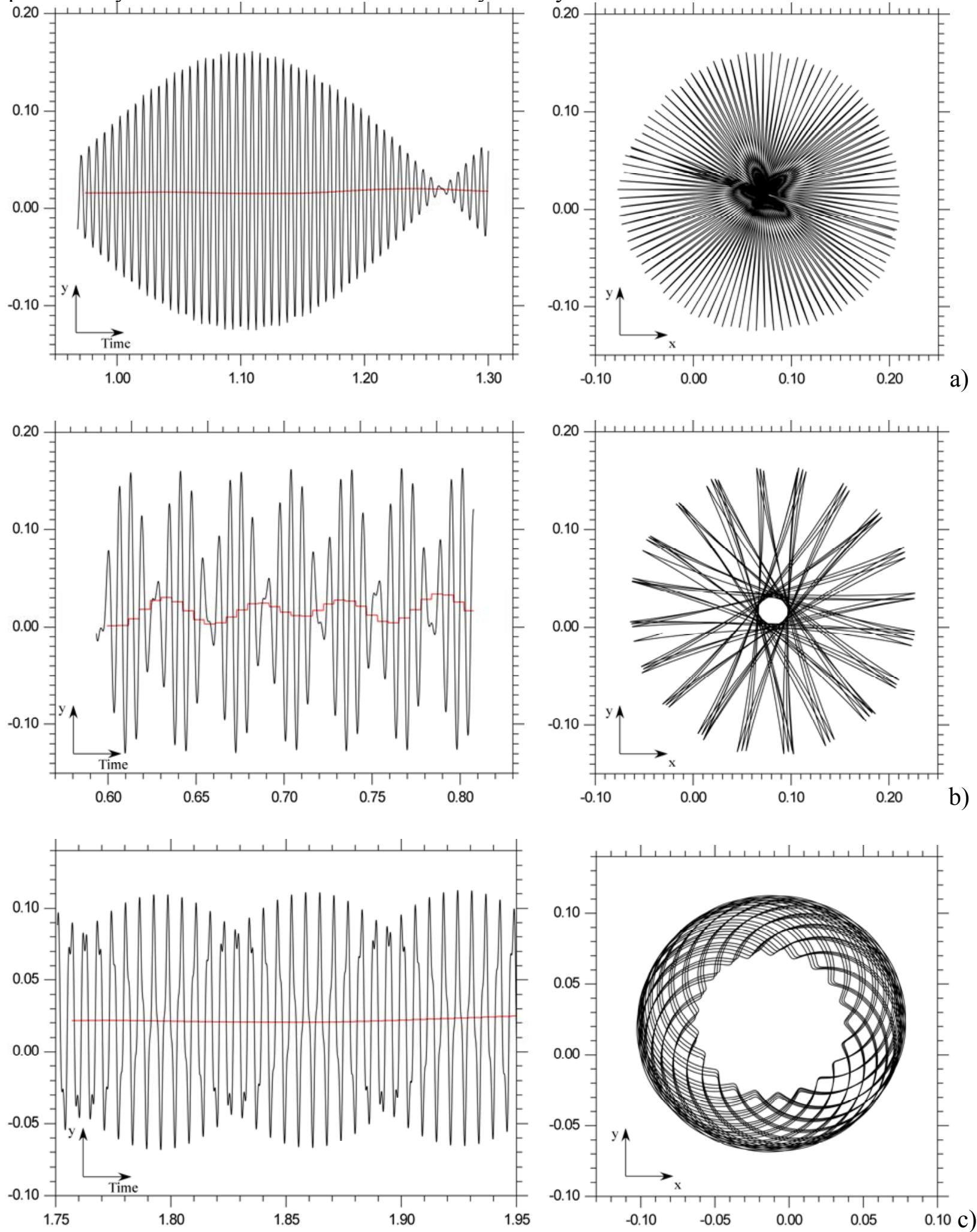

Figure 13: Position of the barycentre of particle aggregates as a function of time for different values of $\psi\left(\right.$ black line $\underline{r}_{G}=\left(1 / N_{\text {partcl }}\right) \sum_{i=1}^{N_{\text {partcl }}} \underline{r}_{i}$, red line $\left.\underline{\underline{r}}_{G}=(\varpi / 2 \pi) \int_{0}^{2 \pi / \pi} \underline{r}_{G} d t\right)$ : a $\left.\left.) \psi=10, \mathrm{~b}\right) \psi=10^{2}, \mathrm{c}\right)$ $\psi=2 \times 10^{3}$ 
When the period of rotation is reduced $\left(\sigma=10^{-1}\right)$, the system enters a new regime in which an asymptotic stationary pattern (in terms of morphological properties) is attained. Ensembles of particles tend to accumulate on structures located in each of the four cavity corners in the xy plane. This results in the emergence of a state with increased symmetry properties, namely a kind of centrally symmetric structure that moves back and forth along the instantaneous direction of the vibration axis as the particles were all rigidly connected with each other (creating the illusion of a solid body not moving at all in a reference system having origin in the barycentre of the particle distribution).

A further decrease in the period of rotation $(\sigma>1)$ leads to a stationary bifurcation responsible for the loss of the centre symmetry and the ensuing formation of a fascinating multi-blade patterns.

These findings confirm the potentialities of these vibrated suspensions as pattern-forming systems of exceptional complexity and reinforce the idea that non-isothermal effects might be effectively exploited in the future to devise new contact-less (vibrations-based) particle control methods for dilute dispersions.

\section{Acknowledgments}

This work has been supported by the UK Space Agency (grant ST/S006354/1) in the framework of the T-PAOLA project.

\section{References}

[01] Melnikov D.E. and Shevtsova V., (2017), Different types of Lagrangian coherent structures formed by solid particles in three-dimensional time-periodic flows, European Physical Journal: Special Topics, 226(6): 1239-1251

[02] Saghir M.Z., Mohamed A., (2018), Effectiveness in incorporating Brownian and thermophoresis effects in modelling convective flow of water- $\mathrm{Al}_{2} \quad \mathrm{O}_{3}$ nanoparticles, International Journal of Numerical Methods for Heat and Fluid Flow 28(1), 47-63.

[03] Balboa-Usabiaga F., Delgado-Buscalioni R., (2011), Particle hydrodynamics: From molecular to colloidal fluids, Particle-Based Methods II - Fundamentals and Applications, pp. 152-163

[04] Gotoda M., Sano T., Kaneko T., and Ueno I., (2015), Evaluation of existence region and formation time of particle accumulation structure (PAS) in half-zone liquid bridge, Eur. Phys. $J$. Special Topics, 224, 299.

[05] Haeri S., (2017), Optimisation of blade type spreaders for powder bed preparation in Additive Manufacturing using DEM simulations, Powder Technology, 321: 94-104.

[06] Delgado-Buscalioni R., Meléndez M., Luis-Hita J., Marqués M. I., Sáenz J. J., (2018), Emergence of collective dynamics of gold nanoparticles in an optical vortex lattice, Phys. Rev. E 98, 062614.

[07] Esmaeeli A., (2005), Phase distribution of bubbly flows under terrestrial and microgravity conditions, Fluid Dynamics and Material Processing, 1(1): 63-80. 
[08] Lappa M., (2016), On the nature, formation and diversity of particulate coherent structures in Microgravity Conditions and their relevance to materials science and problems of Astrophysical interest, Geophysical and Astrophysical Fluid Dynamics, 110(4): 348-386.

[09] Lappa M., (2018), On the transport, segregation and dispersion of heavy and light particles interacting with rising thermal plumes, Physics of Fluids, 30(3), 033302 (23 pages).

[10] Lappa M., Drikakis D., Kokkinakis I., (2017), On the propagation and multiple reflections of a blast wave travelling through a dusty gas in a closed box, Physics of Fluids, 29(3), 033301 (19 pages).

[11] Balvin M., Sohn E., Iracki T., Drazer G., and Frechette J., (2009), Directional Locking and the Role of Irreversible Interactions in Deterministic Hydrodynamics Separations in Microfluidic Devices, Phys. Rev. Lett., 103, 078301

[12] Melnikov D.E., Pushkin D.O., Shevtsova V.M., (2013), Synchronization of finite-size particles by a traveling wave in a cylindrical flow, Phys Fluids, 25 (9), 092108

[13] Gotoda M., Melnikov D. E., Ueno I., and Shevtsova V., (2016), Experimental study on dynamics of coherent structures formed by inertial solid, particles in three-dimensional periodic flows, Chaos, 26, 073106 (11 pages).

[14] Balboa Usabiaga F., Delgado-Buscalioni R., Griffith B.E., Donev A., (2014), Inertial coupling method for particles in an incompressible fluctuating fluid, Computer Methods in Applied Mechanics and Engineering, 269: 139-172.

[15] Haller G. and Sapsis T., (2008), Where do inertial particles go in fluid flows?, Physica D: Nonlinear Phenomena, 237(5), 573-583.

[16] Sapsis T. and Haller G., (2010), Clustering criterion for inertial particles in two-dimensional time-periodic and three-dimensional steady flows, Chaos, 20, 017515.

[17] Schwabe D., Mizev A.I., Udhayasankar M. and Tanaka S., (2007), Formation of dynamic particle accumulation structures in oscillatory thermocapillary flow in liquid bridges, Phys. Fluids, 19(7), 072102.

[18] Pushkin D., Melnikov D., Shevtsova V., (2011), Ordering of Small Particles in OneDimensional Coherent Structures by Time-Periodic Flows, Phys. Rev. Lett., 106, 234501.

[19] Lappa M., (2013), Assessment of the role of axial vorticity in the formation of Particle Accumulation Structures (PAS) in supercritical Marangoni and hybrid thermocapillary-rotationdriven flows, Phys. Fluids, 25(1) 012101 (11 pages).

[20] Lappa M., (2013), On the Existence and Multiplicity of One-dimensional Solid Particle Attractors in Time-dependent Rayleigh-Bénard Convection, Chaos, 23(1), 013105 (9 pages).

[21] Lappa M., (2014a), Stationary Solid Particle Attractors in Standing Waves, Phys. Fluids, 26(1), 013305 (12 pages).

[22] Zhou R., Sobecki C.A., Zhang J., Zhang Y., and Wang C., (2017), Magnetic Control of Lateral Migration of Ellipsoidal Microparticles in Microscale Flows, Phys. Rev. Applied, 8, 024019.

[23] Behjatian A. and Esmaeeli A., (2015), Transient electrohydrodynamics of compound drops, Acta Mechanica, 226(8): 2581-2606

[24] Mialdun A., Ryzhkov I.I., Melnikov D.E., and Shevtsova V., (2008), "Experimental Evidence of Thermal Vibrational Convection in a Nonuniformly Heated Fluid in a Reduced Gravity Environment", Phys. Rev. Lett., 101, 084501. 
[25] Lyubimova T.P., Perminov A.V., Kazimardanov M.G. (2019), Stability of quasi-equilibrium states and supercritical regimes of thermal vibrational convection of a Williamson fluid in zero gravity conditions, Int. J. Heat Mass Transf., 129, 406-414.

[26] Lappa M., (2016), Control of convection patterning and intensity in shallow cavities by harmonic vibrations, Microgravity Science \& Technology, 28(1): 29-39.

[27] Tabakova, S.S. and Zapruanov, Z.D. (1982a): On the hydrodynamic interaction of two spheres oscillating in a viscous fluid. I. Axisymmetrical case. J. Appl. Math. Phys. (ZAMP), vol. 33, pp. 344-357.[27] Tabakova, S.S. and Zapruanov, Z.D. (1982b): On the hydrodynamic interaction of two spheres oscillating in a viscous fluid. II. Three dimensional case. J. Appl. Math. Phys. (ZAMP), vol. 33, pp. 487-502.

[28] Tirumkudulu M., Tripathi A., Acrivos A., (1999), Particle segregation in monodisperse sheared suspensions, Phys. Fluids, 11(3), 507-509

[29] Thomson J.R., Casademunt J., Drolet F. and Vinals J., (1997), Coarsening of solid-liquid mixtures in a random acceleration field, Phys. Fluids, 9(5), 1336-1343.

[30] Voth G. A., Bigger B., Buckley M.R., Losert W., Brenner M.P., Stone H.A., and Gollub J.P., Ordered clusters and dynamical states of particles in a vibrated fluid, Phys. Rev. Lett., 88, 234301 (2002).

[31] Ivanova, A.A.and Kozlov, V.G. (2002): Sand-fluid interface under action of vibration. Fluid Dynamics, vol. 37 (2), pp. 277-293.

[32] Kozlov V.G., Ivanova A.A. and Evesque P., (2006), Block stratification of sedimenting granular matter in a vessel due to vertical vibration, Fluid Dyn. Mater. Process., 2(3), 203-210.

[33] Lappa M., (2013), On the variety of particle accumulation structures under the effect of gjitters, J. Fluid Mech., 726: 160-195.

[34] Lappa M., (2014), The patterning behaviour and accumulation of spherical particles in a vibrated non-isothermal liquid, Phys. Fluids, 26(9), 093301 (22 pages).

[35] Lappa M., (2016), Numerical study into the morphology and formation mechanisms of threedimensional particle structures in vibrated cylindrical cavities with various heating conditions, Physical Review Fluids, 1(6), 064203 (25 pages).

[36] Lappa M., (2017), On the multiplicity and symmetry of particle attractors in confined nonisothermal fluids subjected to inclined vibrations, Int. J. Multiphase Flow, 93: 71-83.

[37] Ndukaife J.C., Kildishev A.V., Nnanna A.G.A., Shalaev V.M. and Wereley S.T., (2016), Long-range and rapid transport of individual nano-objects by a hybrid electrothermoplasmonic nanotweezer, Nature nanotechnology 11 (1), 53.

[38] Monti R., Savino R., Lappa M. (1998); Microgravity sensitivity of typical fluid physics experiment, presented at the 17 th Microgravity Measurements Group Meeting, Cleveland, Ohio, 24-26 March 1998, published in the meeting proceedings in NASA CP-1998-208414, (23): 1-15 (ISSN: 0191-7811).

[39] Monti R., Savino R., Lappa M., (2001), On the convective disturbances induced by g-jitter on the space station, Acta Astronautica, 48 (5-12), 603-615.

[40] Balboa Usabiaga F., PagonabarragaI., and Delgado-Buscalioni R., (2013), Inertial coupling for point particle fluctuating hydrodynamics, J. Comp. Phys., 235, 701-722.

[41] Maxey M. R. and Riley J. J., (1983), Equation of motion for a small rigid sphere in a nonuniform flow, Phys. Fluids, 26, 883-889. 
[42] Kuhlmann H.C. et al. (2014), The JEREMI-Project on thermocapillary convection in liquid bridges. Part A: Overview of Particle Accumulation Structures, Fluid Dyn. Mater. Process., 10 (1), $1-36$.

[43] Lappa, (2011) M., Some considerations about the symmetry and evolution of chaotic Rayleigh-Bénard convection: The flywheel mechanism and the "wind" of turbulence, Comptes Rendus Mécanique, 339: 563-572

[44] Lappa M., (2019), Time reversibility and non deterministic behaviour in oscillatorily sheared suspensions of non-interacting particles at high Reynolds numbers, Computers \& Fluids, 184, 78-90. 